\title{
Preparo e caracterização de filmes comestíveis de quitosana
}

\section{Preparation and characterization chitosan edible films}

\author{
Rodrigo Martins Fráguas ${ }^{*}$, Anderson Assaid Simão ${ }^{1}$, Paula Vieira Faria², Estela de Resende Queiroz ${ }^{1}$, \\ Ênio Nazaré de Oliveira Junior ${ }^{2}$ e Celeste Maria Patto de Abreu ${ }^{1}$ \\ 'Departamento de Química, Universidade Federal de Lavras - UFLA, Lavras, MG, Brasil \\ ${ }^{2}$ Departamento de Química, Biotecnologia e Engenharia de Bioprocessos, Universidade Federal de \\ São João Del Rey - UFSJ, Ouro Branco, MG, Brasil \\ *rodrigofraguas1@hotmail.com
}

\begin{abstract}
Resumo
Este trabalho tem como objetivo a caracterização de três amostras comerciais de quitosana de baixo grau de acetilação, bem como a preparação e caracterização de filmes comestíveis a partir dessas amostras, com vistas a posterior aplicação desses filmes em recobrimento de frutos. As amostras foram caracterizadas pela massa molar, grau de acetilação, análise elementar, espectroscopia de infravermelho e análise térmica, enquanto os filmes pela análise térmica, espectroscopia de infravermelho, sólidos totais e permeabilidade ao vapor de água. As amostras comerciais mostraram diferentes massas molares que variaram de 132 a $245 \mathrm{kDa}$, graus de acetilação de 5,8 a 6,3\% e apresentaram análise elementar próxima a fórmula $\mathrm{C}_{6} \mathrm{H}_{11} \mathrm{O}_{4} \mathrm{~N}$ para a unidade de repetição. Os filmes produzidos apresentaram espessuras de 3,7 a 6,8 $\mu \mathrm{m}$, sólidos totais de 2,0 a $6,3 \mathrm{~g} \mathrm{~m}^{-2}$ e permeabilidade ao vapor de água de $170,1 \mathrm{a} 180,2 \mathrm{~g} \mathrm{dia}^{-1} \mathrm{~m}^{-2}$. Os filmes de quitosana com maior teor de sólidos e menor massa molar foram menos permeáveis ao vapor de água. Nas análises de infravermelho e termogravimetria foram observados, respectivamente, os mesmos grupos funcionais e curvas semelhantes de decomposição, para as amostras e para os filmes produzidos.
\end{abstract}

Palavras-chave: caracterização, filmes comestiveis, quitosana.

\begin{abstract}
The objective of this study is to characterize three commercial samples of chitosan with low degree of acetylation, as well as the preparation and characterization of edible films from these samples, aiming the subsequent application of these films in fruit coatings. The samples were characterized by molar mass, degree of acetylation, elemental analysis, infrared spectroscopy and thermal analysis, while the film was characterized by thermal analysis, infrared spectroscopy, total solids and water vapor permeability. The commercial samples showed different molar masses, which ranged from 132 to $245 \mathrm{kDa}$, degrees of acetylation from 5.8 to $6.3 \%$ and presented an elemental analysis next to the formula $\mathrm{C}_{6} \mathrm{H}_{11} \mathrm{O}_{4} \mathrm{~N}_{\text {, }}$ for the repeating unit. The films produced had thicknesses from 3.7 to $6.8 \mu \mathrm{m}$, total solids from 2.0 to $6.3 \mathrm{~g} \mathrm{~m}^{-2}$ and water vapor permeability from 170.1 to $180.2 \mathrm{~g}$ day $^{-1} \mathrm{~m}^{-2}$. The chitosan films with a higher content of solids and a lower molar mass were less permeable to water vapor. Infrared and thermogravimetric analyses showed the same functional groups and similar decomposition curves, respectively, for the samples and for the films produced.
\end{abstract}

Keywords: characterization, edible films, chitosan.

\section{Introdução}

Os polímeros quitina e quitosana têm sido bastante estudados devido ao elevado potencial de aplicações em indústrias alimentícias, farmacêuticas, cosméticas e na agricultura $^{[1-3]}$. Esses polímeros são constituídos de cadeias lineares dos carboidratos 2-acetamido-2-deoxi-D-glicopiranose e 2-amino-2-deoxi-D-glicopiranose unidos por ligações glicosídicas $\beta$ (1-4) e, podem ser distinguidos por suas solubilidades em solução aquosa de ácido acético 1\%. Quitina, contendo um número $\geq 40 \%$ de N-acetil-D-glicosamina é insolúvel, visto que os polímeros solúveis são chamados de quitosana ${ }^{[4,5]}$.

As aplicações desses compostos em várias áreas, sobretudo a aplicação de quitosana, justifica-se pelo baixo custo de produção, a qual é produzida a partir dos descartes do processamento de crustáceos, fonte bastante abundante, sendo as características dos produtos a base de quitosana dependentes da origem da matéria prima e da metodologia empregada para sua produção ${ }^{[6]}$.

Industrialmente a quitosana é produzida a partir da desacetilação alcalina de quitina (hidrólise alcalina), porém, a quitosana também pode ser obtida a partir da desacetilação enzimática de quitina, processo este investigado em pesquisas laboratoriais $^{[7,8]}$.

A quitosana é uma fibra animal que apresenta propriedades biológicas, como baixa toxicidade, biodegradabilidade, 
biocompatibilidade, além de apresentar efeito antifúngico, antibacteriano e induzir respostas de defesa em frutos tratados ${ }^{[9,10]}$. Devido a essas características e a de fácil formação de géis e filmes, a quitosana tem sido considerada a décadas como um composto de interesse nas mais diversas áreas.

Revestimentos à base de quitosana apresentam potencial para controlar perdas causadas por podridões pós-colheita e para prolongar o tempo de armazenamento de frutos. Vários produtos vegetais como tomates ${ }^{[10]}$, pêssegos ${ }^{[11]}$, maçãs ${ }^{[12]}$, cajus $^{[1]}$, mamão ${ }^{[13]}$ e lichias ${ }^{[14]}$, entre outros frutos, têm o tempo de armazenamento estendido quando revestidos com quitosana, devido à formação de um filme semipermeável que regula as trocas gasosas e reduz as perdas por transpiração, retardando o amadurecimento dos frutos. Para a maioria destes estudos os filmes produzidos são obtidos de quitosanas de alto grau de acetilação. Este estudo se diferencia por caracterizar amostras comerciais de quitosanas de baixo grau de acetilação e por produzir e caracterizar filmes destas quitosanas.

Neste contexto, o objetivo deste trabalho foi caracterizar amostras comerciais de quitosanas de baixo grau de acetilação, bem como a preparação e caracterização de filmes comestíveis dessas amostras.

\section{Experimental}

As amostras de quitosana (nome comercial Chitoclear ${ }^{\circledR}$ ) foram gentilmente cedidas pela Primex ehf da Islândia, em três lotes diferentes: 3493, 3529 e 3142, e codificadas, respectivamente, como A, B e C. Na Tabela 1 estão descritos os valores das viscosidades das amostras fornecidas pelo fabricante.

\subsection{Determinação da massa molar média absoluta por cromatografia de permeação em gel}

Para a determinação da massa molar média absoluta as amostras de quitosana foram dissolvidas em tampão acetato de sódio pH 4 na proporção 1:100 (quitosana/tampão, m/v), filtradas em filtros $0,45 \mu \mathrm{m}$ e, em seguida, submetidas a cromatografia de permeação em gel. A análise foi realizada utilizando duas colunas em série Ultrahydrogel ${ }^{\mathrm{TM}}$ Linear 7,8 x 300 mm, um injetor Viscotek GPCmax VE 2001 Solvent/SAMPLE MODULE, detector Viscotek TDA302 Triple Detector Array, tendo como fase móvel tampão acetato de sódio $\mathrm{pH} 4$. O volume injetado foi de $100 \mu \mathrm{L}$ a uma vazão de $0,8 \mathrm{~mL} \mathrm{~min}^{-1}$ e o equipamento é dotado dos seguintes detectores: índice de refração (IR), viscosidade (DP) e espalhamento de luz (RALS).

Tabela 1. Viscosidade, grau de acetilação (GA) e massa molar absoluta (Mw) de amostras de quitosana.

\begin{tabular}{cccc}
\hline Amostra & $\begin{array}{c}\text { Viscosidade } \\
(\mathbf{m P a} \times \mathbf{s})^{\mathbf{1}}\end{array}$ & GA (\%) & Mw (kDa) \\
\hline A & 5 & $5,9 \pm 0,1$ & $132 \pm 1,0$ \\
B & 520 & $5,8 \pm 0,1$ & $228 \pm 9,0$ \\
C & 1.300 & $6,3 \pm 0,1$ & $245 \pm 2,0$ \\
\hline
\end{tabular}

${ }^{1}$ Informação cedida pelo fabricante (Primex ehf).

\subsection{Determinação do grau de acetilação}

O grau de acetilação (GA) das amostras de quitosana foi determinado pelo método de titulação potenciométrica segundo metodologia descrita por Raymond et al. ${ }^{[15]}$. As amostras foram dissolvidas em $\mathrm{HCl} 0,1 \mathrm{~mol} \mathrm{~L}^{-1}$ na proporção 1:100 (quitosa $/ \mathrm{HCl}, \mathrm{m} / \mathrm{v}$ ), e titulou-se com solução padrão de $\mathrm{NaOH}$ 0,1 mol L-1 sob agitação contínua. Os valores de GA foram calculados através da Equação 1:

$$
G A=\frac{\left(V_{\mathrm{NaOH}} \times M_{q u}\right) \times 100}{\left(1000 \times M_{\mathrm{NaOH}} \times m_{q u}\right)}
$$

onde, $\mathrm{GA}=$ grau de acetilação de quitosana $(\%) ; \mathrm{m}_{\mathrm{qu}}=$ massa de amostra de quitosana (g); $\mathrm{M}_{\mathrm{qu}}=$ massa molar da unidade de glicosamina $\left(161 \mathrm{~g} \mathrm{~mol}^{-1}\right) ; \mathrm{V}_{\mathrm{NaOH}}^{\text {u }}=$ volume da solução de $\mathrm{NaOH}\left(0,1 \mathrm{~mol} \mathrm{~L}^{-1}\right)$ usado para neutralizar os grupos amino livres protonados $(\mathrm{mL})$ e $\mathrm{M}_{\mathrm{NaOH}}=$ concentração molar real da solução de $\mathrm{NaOH}\left(\mathrm{mol} \mathrm{L}^{-1}\right)$.

\subsection{Análise elementar}

Para a determinação da análise elementar $2 \mathrm{mg}$ de cada amostra de quitosana foram secas em estufa a $103 \pm 2{ }^{\circ} \mathrm{C}$ e posteriormente acondicionadas em analisador elementar Vario Micro Cube. Desta forma, foram obtidos os teores em nitrogênio, carbono, hidrogênio, enxofre e, por diferença, o teor em oxigênio. A temperatura do tubo de combustão foi de $1200^{\circ} \mathrm{C}$ e a temperatura do tubo de redução de $850{ }^{\circ} \mathrm{C}$, sendo utilizado o hélio como gás de arraste e o oxigênio como gás oxidante.

\subsection{Produção dos filmes de quitosana}

Para o preparo dos filmes das amostras de quitosana seguiu-se o procedimento descrito por Yoshida et al. ${ }^{[16]}$. Os filmes foram preparados dissolvendo as amostras de quitosana em solução de ácido acético $1 \%$ nas concentrações $0,25,0,5,1,0$ e $1,5 \%$. As soluções foram homogeneizadas em agitador magnético (Fisatom, modelo 711) a temperatura ambiente por 24 horas até completa dissolução. Em seguida, foram filtradas em filtro de $11 \mu \mathrm{m}$ sob vácuo e $10 \mathrm{~mL}$ do filtrado foi adicionado em placas de Petri de poliestireno de $8 \mathrm{~cm}$ de diâmetro. Os filmes foram secos a temperatura ambiente.

\subsection{Espectroscopia na região do infravermelho}

A análise de infravermelho foi realizada nas amostras de quitosana e nos filmes produzidos na concentração de $1 \%$. Aproximadamente 1,5 mg das amostras foram secas em estufa a vácuo por 15 horas a $60{ }^{\circ} \mathrm{C}$. Após este período, $100 \mathrm{mg}$ de $\mathrm{KBr}$ foram adicionados e a mistura homogeneizada em almofariz de ágata. As pastilhas foram preparadas e deixadas em estufa a vácuo a $110^{\circ} \mathrm{C}$ por 20 horas. Os espectros de infravermelho foram registrados em um espectrofotômetro Bomem-Michelson FT-IR, MB 102.

\subsection{Análise térmica}

A análise térmica foi feita nas amostras de quitosana e nos filmes produzidos na concentração de $1 \%$. As curvas termogravimétricas foram obtidas em um módulo 
termogravimétrico TGA 2050, acoplado a um analisador térmico TA 2000 da TA instruments. A atmosfera empregada foi de $\mathrm{N}_{2}$ st, com razão de aquecimento de $10^{\circ} \mathrm{C} \mathrm{min}{ }^{-1}$ até a temperatura de $400{ }^{\circ} \mathrm{C}$ e o fluxo de gás de $30 \mathrm{~mL} \mathrm{~min}^{-1}$.

\subsection{Espessura do filme}

As espessuras dos filmes das amostras de quitosana A, B e C preparados nas concentrações $0,5,1$ e $1,5 \%$, foram medidas através de micrômetro (Mitutoyo Mfg. Co. Ltd., Kawasakishi, Kanagawa, Japão). As medidas foram realizadas aleatoriamente em cinco posições diferentes dos filmes e a espessura do filme foi à média dessas medidas.

\subsection{Sólidos totais}

Os filmes produzidos a partir das amostras de quitosana A, B e C preparados nas concentrações $0,5,1$ e 1,5\%, foram pesados em balança analítica e as massas obtidas foram divididas pela área da placa de petri de $8 \mathrm{~cm}$ de diâmetro, que é de $0,005 \mathrm{~m}^{2}$. Os teores de sólidos totais dos filmes em $\mathrm{g} \times \mathrm{m}^{-2}$ foram determinados dividindo a massa obtida para cada filme em gramas pela área de $0,005 \mathrm{~m}^{2}$.

\subsection{Cinética de permeabilidade ao vapor de água}

A permeabilidade ao vapor de água dos filmes das amostras de quitosana $\mathrm{A}, \mathrm{B}$ e $\mathrm{C}$ preparados nas concentrações $0,5,1$ e $1,5 \%$, foram determinadas pelo método gravimétrico padrão E96-95 ${ }^{[17]}$. Em frascos de $50 \mathrm{~mL}$ foram colocados $10 \mathrm{~g}$ de sílica gel azul (Synth) e os mesmos foram lacrados com os filmes de quitosana e colocados dentro de um dessecador no qual continha $200 \mathrm{~mL}$ de solução salina de cloreto de cálcio $10 \%(\mathrm{~m} / \mathrm{v})$. Após este procedimento foi feito vácuo dentro do dessecador e o mesmo foi levado a uma BOD (Novatecnica, NT 705) a temperatura de $25^{\circ} \mathrm{C}$ e durante cinco dias as amostras eram retiradas e pesadas e um novo vácuo realizado.

\subsection{Análise estatística}

A análise estatística foi realizada no programa Origin Pro $8 \mathrm{e}$ as diferenças entre as médias obtidas a partir de triplicatas foram analisadas pelo teste de Tukey $(\mathrm{p} \leq 0,05)$.

\section{Resultados e Discussão}

\subsection{Massa molar e grau de acetilação}

Os resultados das massas molares e dos GA das amostras de quitosana estão apresentados na Tabela 1. As massas molares médias obtidas pela técnica de cromatografia de permeação em gel para três amostras de quitosana codificadas como A, B e C, foram respectivamente 132, 228 e $245 \mathrm{kDa}$. Assim como era esperado, com o aumento dos valores das viscosidades das amostras de quitosanas $\mathrm{A}, \mathrm{B} \mathrm{e} \mathrm{C}$ fornecidos pelo fabricante, respectivamente 5,520 e $1.300 \mathrm{mPa} \times \mathrm{s}$, também houve aumento dos valores das massas molares médias.

Apesar da tendência de aumento observado nos dois métodos, viscosimétrico e cromatográfico, não se observou um aumento proporcional entre os valores, tendo em vista que os princípios de detecção dos dois métodos são distintos. Um método se baseia na determinação da viscosidade (viscosimétrico) e o método de cromatografia de permeação em gel avançado se baseia na combinação de três detectores (índice de refração, viscosidade e espalhamento de luz) através do qual se determina o valor da massa molar média.

Os GA obtidos pela técnica de titulação potenciométrica para as três amostras de quitosana codificadas como A, B e C, foram respectivamente 5,9, 5,8 e 6,3\%. O GA obtido pelo método de titulação potenciométrica é bastante confiável quando as soluções de $\mathrm{HCl}$ e $\mathrm{NaOH} 0,1 \mathrm{~mol} \mathrm{~L}^{-1}$ são padronizadas e quando as amostras de quitosana são totalmente solúveis na solução de $\mathrm{HCl}$ e ambas situações foram observadas neste estudo.

Raymond et al. ${ }^{[15]}$ observaram que a titulação potenciométrica é um método indicado para amostras de quitosana com baixos valores de GA conforme foi o caso deste estudo. Oliveira et al. ${ }^{[18]}$ comparando os resultados de GA obtidos por titulação potenciométrica e por RMN ${ }^{1} \mathrm{H}$ (ressonância magnética nuclear de próton) observaram que não houve diferença entre estes métodos para quitosana com GA que variaram entre 8 e $22 \%$. Desta maneira, o método de titulação potenciométrica mostra-se um método simples, barato e bastante confiável na determinação do GA de quitosana, desde que se tomem os cuidados anteriormente citados.

\subsection{Análise elementar}

Os dados de análise elementar apresentados na Tabela 2 são próximos da fórmula $\mathrm{C}_{6} \mathrm{H}_{11} \mathrm{O}_{4} \mathrm{~N}$ para a unidade de repetição, quando se desconta o teor de água presente na amostra.

A hidratação se deve ao fato de que a quitosana tem forte afinidade pela água e, no estado sólido, as macromoléculas podem apresentar estruturas distorcidas, que são facilmente hidratadas ${ }^{[19]}$. Em adição, deve-se considerar que a análise elementar é um método conveniente para análise de quitosana somente na ausência de proteína residual ${ }^{[7,20]}$.

\subsection{Infravermelho}

Os espectros de absorção no infravermelho das amostras de quitosana e do filme produzido a partir da amostra A na concentração 1\% são apresentados na Figura 1. Optou-se por apresentar somente um resultado para os filmes, uma vez que os espectros de absorção no infravermelho para os demais filmes foram semelhantes.

As principais bandas observadas nos espectros das amostras de quitosana e do filme foram: banda de estiramento axial de $\mathrm{OH}$, atribuída ao grupo hidroxila presente na quitosana entre $3440 \mathrm{a} 3480 \mathrm{~cm}^{-1}$, a qual aparece sobreposta à banda de estiramento $\mathrm{N}-\mathrm{H}$; as bandas na região de $2854 \mathrm{~cm}^{-1}$ são atribuídas aos grupos $\mathrm{CH}_{2}$ das piranoses, são observadas sobreposições de bandas dos grupos amidas e grupos $\mathrm{OH}$ das piranoses nas regiões entre 1661 a $1671 \mathrm{~cm}^{-1}$; deformação

Tabela 2. Análise elementar das amostras de quitosana.

\begin{tabular}{crcc}
\hline \multirow{2}{*}{ Amostra } & \multicolumn{3}{c}{ Composição (\%) } \\
\cline { 2 - 4 } & \multicolumn{1}{c}{$\mathbf{N}^{\mathbf{1}}$} & \multicolumn{1}{c}{$\mathbf{C}^{\mathbf{2}}$} & \multicolumn{1}{c}{$\mathbf{H}^{\mathbf{3}}$} \\
\hline $\mathrm{A}$ & $8,1 \pm 0,8$ & $38,4 \pm 3,7$ & $6,9 \pm 0,5$ \\
$\mathrm{~B}$ & $10,3 \pm 0,5$ & $38,0 \pm 3,3$ & $6,9 \pm 0,4$ \\
$\mathrm{C}$ & $9,7 \pm 0,9$ & $37,2 \pm 2,2$ & $6,5 \pm 0,5$ \\
\hline
\end{tabular}

${ }^{1}$ Nitrogênio. ${ }^{2}$ Carbono. ${ }^{3}$ Hidrogênio. 


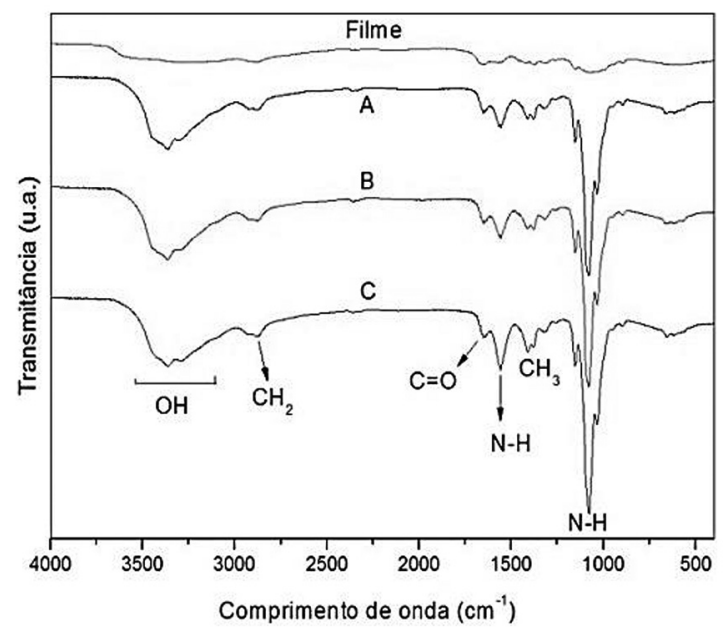

Figura 1. Espectros de infravermelho das amostras de quitosana A, $\mathrm{B} \mathrm{e} \mathrm{C}$ e do filme produzido a partir da amostra $\mathrm{A}$ na concentração de $1 \%$.

angular de $\mathrm{N}-\mathrm{H}$ (entre 1583 a $1594 \mathrm{~cm}^{-1}$ ), o modo referente às amidas é observado na região de $1500 \mathrm{~cm}^{-1}$ e entre $1200-800 \mathrm{~cm}^{-1}$ as vibrações estão associadas às ligações químicas das piranoses.

As bandas consideravelmente fortes para a quitosana são observadas na região entre 1700 e $1300 \mathrm{~cm}^{-1}$ que são especialmente características deste biopolímero. As bandas na região $1640 \mathrm{~cm}^{-1}$, são atribuídas à deformação axial $\mathrm{C}=\mathrm{O}$ da carbonila denominada $v \mathrm{C}=\mathrm{O}$, do grupo acetamida, a qual corresponde à parte acetilada da quitosana. As bandas na faixa de $1500 \mathrm{~cm}^{-1}$ correspondem a vibração N-H no plano denominada $v \mathrm{~N}-\mathrm{H}$. As bandas por volta de $1300 \mathrm{e} 1400 \mathrm{~cm}^{-1}$ corresponde à deformação angular simétrica do grupo $\mathrm{CH}_{3}$.

Nos espectros vibracionais dos filmes de quitosana, percebeu-se que as bandas características do bíopolimero se mantiveram, no entanto, foi possível observar uma diminuição das intensidades de acordo com a concentração da quantidade de quitosana empregada na elaboração dos filmes.

Todas as bandas características são muito semelhantes aquelas relatadas na literatura ${ }^{[20,21]} \mathrm{e}$ estão presentes em todas as amostras investigadas e no filme, mostrando que todas apresentam basicamente os mesmos grupos funcionais.

\subsection{Análise termogravimétrica}

As curvas de termogravimetria das amostras de quitosana e do filme produzido a partir da amostra A na concentração $1 \%$ são mostrados na Figura 2. Optou-se mais uma vez por apresentar somente um resultado para os filmes visto que, as curvas termogravimétricas para os demais filmes foram semelhantes.

Os termogramas das quitosanas e do filme apresentaram somente duas etapas de decomposição. No primeiro estágio, próximo a $100^{\circ} \mathrm{C}$ a primeira perda esta relacionada à perda de moléculas de água que estariam ligados aos grupamentos amino e hidroxila, através de pontes de hidrogênio, então tal evento está relacionado tanto com as interações polímero-água, quanto com a capacidade do polímero reter água.

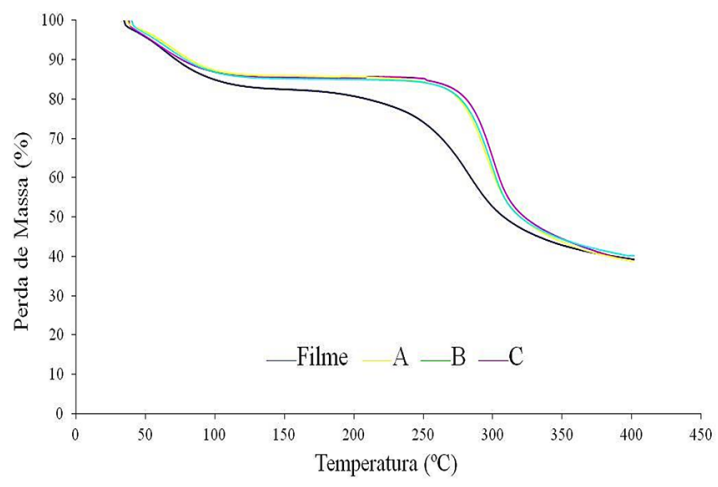

Figura 2. Termograma das amostras de quitosana A, B e C e do filme produzido a partir da amostra A na concentração de $1 \%$.

O segundo estágio, cujo patamar final encontra-se indefinido, está relacionada a perda de massa atribuída ao complexo processo de decomposição que tem início através da quebra aleatória de ligações glicosídicas, seguida da decomposição das unidades acetiladas e desacetiladas do polímero, porém, como a atmosfera é a de um gás inerte, esta perda de massa está relacionada mais ao efeito térmico propriamente dito do que ao efeito reacional[ ${ }^{[22]}$.

Para a amostra do filme nota-se que o padrão da curva se manteve semelhante aos das amostras com uma menor resistência ao calor, tal fato pode ser relacionado com a menor concentração de quitosana, visto que o filme foi preparado usando apenas $1 \%$ de amostra. É importante frisar também que os materiais analisados, em atmosfera inerte $\left(\mathrm{N}_{2}\right)$, apresentaram boa estabilidade térmica até temperaturas próximas de $300{ }^{\circ} \mathrm{C}^{[23]}$.

\subsection{Filmes de quitosana}

As concentrações empregadas para as amostras de quitosana $(0,5,1,0$ e $1,5 \%)$ revelaram a formação de filmes homogêneos, finos, transparentes e flexíveis. Exceto para a concentração $0,25 \%$ que formou filmes extremamente frágeis que proporcionaram grandes dificuldades de manuseio, desta maneira, foram analisados somente os filmes obtidos a partir da concentração $0,5 \%$.

Observou-se aumento significativo ( $\mathrm{p} \leq 0,05)$ da espessura dos filmes com o aumento da concentração da solução de quitosana para todas as amostras (Tabela 3), entretanto, pode-se observar que com o aumento das massas molares das amostras de quitosana (132 a $245 \mathrm{kDa}$ ), apesar de se observar uma tendência de aumento das espessuras dos filmes, não houve diferença significativa $(p \leq 0,05)$ entre os valores das espessuras dos filmes produzidos nas mesmas concentrações.

Os filmes produzidos neste estudo apresentaram espessuras que variaram de 3,7 a 6,8 $\mu \mathrm{m}$ (Tabela 3 ), e foram muito menores se comparados aos resultados encontrados por Liu et al. ${ }^{[24]}(28,1 \pm 1,1$ a $28,7 \pm 1,1 \mu \mathrm{m})$ e Peng e Li[i ${ }^{[23]}$ $(77,0 \pm 7,0$ a $105,0 \pm 2,0 \mu \mathrm{m})$. Entretanto, as espessuras dos filmes produzidos por Leceta et al. ${ }^{[25]}$ variaram de $0,060 \pm 0,007$ a $0,068 \pm 0,006 \mu \mathrm{m}$ e se comparados com os filmes produzidos neste trabalho, foram aproximadamente 100 vezes mais finos. 
Tabela 3. Propriedades das amostras de quitosana e de seus respectivos filmes produzidos.

\begin{tabular}{|c|c|c|c|c|c|}
\hline \multirow[b]{2}{*}{ Amostra } & \multicolumn{2}{|c|}{ Propriedades das quitosanas } & \multicolumn{3}{|c|}{ Propriedades dos filmes } \\
\hline & Massa molar (kDa) & Concentração \% (m/v) & Espessura $(\mu \mathrm{m})$ & $\begin{array}{c}\text { Sólidos totais } \\
\left(\mathrm{g} \times \mathbf{m}^{-2}\right)\end{array}$ & $\begin{array}{c}\text { PVA }^{1} \\
\left(\mathrm{~g} \times \operatorname{dia}^{-1} \times \mathrm{m}^{-2}\right)\end{array}$ \\
\hline \multirow{3}{*}{ A } & \multirow{3}{*}{132} & 0,5 & $3,7^{\mathrm{a}}$ & $2,0^{\mathrm{c}}$ & $176,6^{\mathrm{f}}$ \\
\hline & & 1,0 & $4,5^{\mathrm{a}}$ & $4,0^{\mathrm{de}}$ & $173,2^{\mathrm{f}}$ \\
\hline & & 1,5 & $6,0^{\mathrm{ab}}$ & $7,3^{\mathrm{e}}$ & $170,2^{\mathrm{f}}$ \\
\hline \multirow{3}{*}{ B } & \multirow{3}{*}{228} & 0,5 & $4,6^{\mathrm{a}}$ & $2,0^{\mathrm{c}}$ & $179,6^{\mathrm{fg}}$ \\
\hline & & 1,0 & $6,0^{\mathrm{ab}}$ & $3,5^{\mathrm{cd}}$ & $176,8^{\mathrm{f}}$ \\
\hline & & 1,5 & $6,1^{\mathrm{ab}}$ & $6,3^{\mathrm{e}}$ & $170,1^{\mathrm{f}}$ \\
\hline \multirow{3}{*}{$\mathrm{C}$} & \multirow{3}{*}{245} & 0,5 & $4,5^{\mathrm{a}}$ & $2,2^{\mathrm{c}}$ & $180,2^{\text {fg }}$ \\
\hline & & 1,0 & $5,3^{\mathrm{a}}$ & $4,3^{\mathrm{de}}$ & $174,5^{\mathrm{f}}$ \\
\hline & & 1,5 & $6,8^{\mathrm{ab}}$ & $4,8^{\mathrm{de}}$ & $175,3^{\mathrm{f}}$ \\
\hline
\end{tabular}

${ }^{1}$ PVA: permeabilidade ao vapor de água. ${ }^{\text {a-f }}$ Médias na mesma coluna com diferentes sobrescritos diferem significativamente a p $\leq 0,05$ de acordo com o teste de Tukey.

Essas diferenças de espessuras dos filmes de quitosana observadas neste estudo e os de outros trabalhos se justificam pelo fato de que as condições de preparo dos filmes de quitosana nem sempre são as mesmas, dentre as quais, pode-se citar a concentração da solução de quitosana, volume de solução e amostras de quitosana com diferentes massas molares.

A medida que o volume da solução utilizada foi mantida constante $(10 \mathrm{~mL})$, os filmes obtidos tinham seu teor de sólidos totais por unidade de área aumentado com o aumento das concentrações das soluções (Tabela 3). Exceto para a amostra C, cujos teores de sólidos totais nas concentrações 1,0 e $1,5 \%$ não variaram significativamente $(\mathrm{p} \leq 0,05)$.

Isto se deve ao fato da amostra $\mathrm{C}$ ser muito viscosa, $1.300 \mathrm{mPa} \times \mathrm{s}$, e quanto maior a concentração, maior a retenção de amostra durante a filtração em filtro de vidro sinterizado, que no caso da concentração de $1,5 \%$ a retenção foi de $10 \%$. O filme com maior teor de sólidos por área que foi de 7,3 $\mathrm{g} \times \mathrm{m}^{-2}$ foi obtido a partir da amostra A de menor viscosidade ( $5 \mathrm{mPa} \times \mathrm{s})$, o que justifica o maior teor de sólidos devido não ter havido retenção de sólidos durante a filtração.

Não foi observado a formação de bolhas de ar incorporadas as matrizes dos filmes com maior massa por área como descrito por Yoshida et al. ${ }^{[16]}$.

Os resultados de permeabilidade ao vapor de água dos filmes obtidos a partir das amostras de quitosana (Tabela 3) mostraram que houve uma tendência de diminuição da permeabilidade com o aumento dos sólidos totais por metro quadrado de filme para uma mesma amostra de quitosana. Para a amostra A (viscosidade $5 \mathrm{mPa} \times \mathrm{s}$ ), a permeabilidade ao vapor de água dos filmes de quitosana produzidos a partir das concentrações $0,5,1,0$ e 1,5\% foram respectivamente, $176,6,173,2$ e $170,2 \mathrm{~g} \times \mathrm{dia}^{-1} \times \mathrm{m}^{-2}$, e se mostraram menos permeáveis ao vapor de água durante cinco dias de análise comparados aos outros filmes produzidos a partir das amostras B e C.

Comparando-se os valores de permeabilidade ao vapor de água dos filmes produzidos a partir da solução de concentração $1,5 \%$, pode-se notar que os valores obtidos para as amostras A e B não apresentaram diferença significativa $(p \leq 0,05)$, sendo respectivamente, 170,2 e $170,1 \mathrm{~g} \times \mathrm{dia}^{-1} \times \mathrm{m}^{-2}$, porém, estes foram significativamente diferentes do valor de permeabilidade do filme obtido a partir da amostra $\mathrm{C}$ que foi de $175,3 \mathrm{~g} \times \mathrm{dia}^{-1} \times \mathrm{m}^{-2}$.

Esse resultado está de acordo com o estudo realizado por Yoshida et al. ${ }^{[16]}$, que também observaram redução da permeabilidade ao vapor de água de 414,63 para $180,09 \mathrm{~g} \times \mathrm{dia}^{-1} \times \mathrm{m}^{-2}$ em filmes produzidos a partir de amostras de quitosana com massas molares de 235 e 13,7 kDa, respectivamente. No presente estudo, não foi observada uma grande diferença da PVA entre os filmes de quitosana produzidos a partir das amostras com massas molares entre 132 e $245 \mathrm{kDa}$, apesar de observarmos uma tendência de diminuição da permeabilidade com a diminuição da massa molar.

Comparando-se os resultados deste estudo com os obtidos por Yoshida et al. ${ }^{[16]}$, pode-se observar que o filme de quitosana $(1 \% \mathrm{~m} / \mathrm{v})$ produzido neste estudo a partir da amostra de massa molar $245 \mathrm{kDa}$, apresentou uma PVA de $174,5 \mathrm{~g} \times \mathrm{dia}^{-1} \times \mathrm{m}^{-2}$, enquanto que o filme de quitosana $(1 \% \mathrm{~m} / \mathrm{v})$ produzido por Yoshida et al. ${ }^{[16]}$ usando uma amostra de massa molar de $235 \mathrm{kDa}$, apresentou-se aproximadamente duas vezes mais permeável ao vapor de água, com um valor de 414,63 $\mathrm{g} \times \mathrm{dia}^{-1} \times \mathrm{m}^{-2}$.

Os resultados obtidos no presente estudo mostram que filmes de quitosana com menor massa molar são menos permeáveis ao vapor de água e essa propriedade influencia diretamente na permeabilidade de revestimentos a base de quitosana com vistas à posterior aplicação desses filmes em recobrimento de frutos.

\section{Conclusões}

As amostras comerciais de quitosana analisadas apresentam diferentes massas molares e graus de acetilação e análise elementar próxima a fórmula $\mathrm{C}_{6} \mathrm{H}_{11} \mathrm{O}_{4} \mathrm{~N}$ para a unidade de repetição. As bandas observadas no infravermelho revelam que todas as quitosanas e os filmes produzidos apresentam basicamente os mesmos grupos funcionais, assim como a análise térmica que mostra curvas semelhantes de decomposição para as amostras e os filmes.

Os filmes de quitosana produzidos a partir da concentração 0,5\% mostraram-se homogêneos, finos, transparentes e flexíveis. As espessuras dos filmes não variaram com as 
massas molares, entretanto, observou-se aumento das espessuras destes com o aumento das concentrações das soluções de quitosana de 0,5 a 1,5\% (m/v). Os filmes com maior teor de sólidos e menor massa molar foram menos permeáveis ao vapor de água.

Os resultados deste estudo mostram que quando se deseja produzir um filme de quitosana menos permeável ao vapor de água é necessário à utilização de amostras de quitosana com baixas massas molares em soluções mais concentradas.

\section{Referências}

1. Oliveira, E. N., Jr., El Gueddari, N. E., Moerschbacher, B. M., \& Franco, T. T. (2012). Growth rate inhibition of phytopathogenic fungi by characterized chitosans. Brazilian Journal of Microbiology, 43(2), 800-809. http://dx.doi. org/10.1590/S1517-83822012000200046. PMid:24031893.

2. Soares, T. C., Sales, F. M. S., Santos, J. W., \& Carvalho, M. F. C. (2014). Quitosana e fitorreguladores na indução da organogênese direta em cultivar de algodão colorido. Revista Brasileira de Engenharia Agrícola e Ambiental, 18(8), 839-843. http://dx.doi.org/10.1590/1807-1929/agriambi.v18n08p839-843.

3. Freddo, A. R., Mazaro, S. M., Brun, E. J., \& Wagner, A. W., Jr. (2014). Quitosana como fungistático no crescimento micelial de Rhizoctonia solani Kuhn. Ciência Rural, 44(1), 1-4. http:// dx.doi.org/10.1590/S0103-84782014000100001.

4. Assis, O. B. G., \& Silva, V. L. (2003). Caracterização estrutural e da capacidade de absorção de água em filmes finos de quitosana processados em diversas concentrações. Polímeros: Ciência e Tecnologia, 13(4), 223-228. http://dx.doi.org/10.1590/ S0104-14282003000400006.

5. Vieira, E. F. S., Cestari, A. R., Chagas, R. A., \& Cortes, G. K. R. (2014). Obtenção e caracterização de matriz apropriada para sistemas de liberação prolongada: estudos de liberação dos herbicidas atrazina e diuron. Quimica Nova, 37(3), 398-403. http://dx.doi.org/10.5935/0100-4042.20140074.

6. Oliveira, B. S., \& Nunes, M. L.(2011). Avaliação de quitosana de caranguejo-uçá (Ucides cordatus) como biofilme protetor em caju. Scientia Plena, 7(4), 041501.

7. Santos, J. E., Soares, J. P., Dockal, E. R., Campana, S. P., Fo., \& Cavalheiro, E. T. G. (2003). Caracterização de quitosanas comerciais de diferentes origens. Polimeros: Ciência e Tecnologia, 13(4), 242-249. http://dx.doi.org/10.1590/S010414282003000400009

8. Gomes, L. P., Oliveira, C. I. R., Silva, M. C., Andrade, C. T., Del Aguila, E. M., Silva, J. T., \& Paschoalin, V. M. F. (2010). Purificação e caracterização da quitinase de uva (Vitis vinífera L. cv Red Globe) para a produção de quitosana a partir de quitina de camarão. Quimica Nova, 33(9), 1882-1886. http:// dx.doi.org/10.1590/S0100-40422010000900012.

9. Dutta, P. K., Tripathi, S., Mehrotra, G. K., \& Dutta, J. (2009). Perspectives for chitosan based antimicrobial films in food applications. Food Chemistry, 114(4), 1173-1182. http://dx.doi. org/10.1016/j.foodchem.2008.11.047.

10. Costa, T. L. E., Oliveira, T. A., Santos, F. K. G., Aroucha, M. M., \& Leite, R. H. L. (2012). Avaliação de coberturas comestíveis compostas por quitosana e argila no revestimento em tomates sob refrigeração pelo método dipping. Revista Verde de Agroecologia e Desenvolvimento Sustentável, 7(5), 12-19.

11. Santos, C. A. A., Castro, J. V., Picoli, A. A., \& Rolim, G. S. (2008). Uso de quitosana e embalagem plástica na conservação pós-colheita de pêssegos 'douradão. Revista Brasileira de Fruticultura, 30(1), 88-93. http://dx.doi.org/10.1590/S010029452008000100017.
12. Botelho, R. V., Maia, A. J., Rickli, E. H., Leite, C. D., \& Faria, C. M. D. R.(2010). Quitosana no controle de Penicillium sp na pós-colheita de maçãs. Revista Brasileira de Agroecologia. 5(2), 200-206.

13. Besinela Júnior, E., Monarim, M. M. S., Camargo, M., Mahl, C. R. A., Simões, M. R., \& Silva, C. F.(2010). Efeito de diferentes biopolímeros no revestimento de mamão (Carica Papaya L) minimamente processado. Revista Varia Scientia Agrárias, 1(1), 131-142.

14. Guimarães, J. E. R., Morgado, C. M. A., Galati, V. C., Marques, K. M., \& Mattiuz, B. H. (2013). Ácido cítrico e quitosana na conservação de lichias 'Bengal. Revista Brasileira de Fruticultura, 35(3), 730-737. http://dx.doi.org/10.1590/S010029452013000300009.

15. Raymond, L., Morin, F. G., \& Marchessault, R. H. (1993). Degree of deacetylation of chitosan using conductometric titration and solid-state NMR. Carbohydrate Research, 246(1), 331-336. http://dx.doi.org/10.1016/0008-6215(93)84044-7.

16. Yoshida, C. M. P., Oliveira, E. N., Jr., \& Franco, T. T. (2009). Chitosan tailor-made films: the effects of additives on barrier and mechanical properties. Packing Technology and Science, 22(3), 161-170. http://dx.doi.org/10.1002/pts.839.

17. American Society for Testing and Materials - ASTM. (1995). ASTM standard test methods for determining gas permeability characteristics of plastic film and sheeting. Philadelphia: ASTM.

18. Oliveira, A. M., Franco, T. T., \& Oliveira-Junior, E. N. (2014). Physicochemical Characterization of Thermally Treated Chitosans and Chitosans Obtained by Alkaline Deacetylation. International Journal of Polymer Science, 9. http://dx.doi. org/10.1155/2014/853572.

19. Rinaudo, M., Desbrières, J., Le Dung, P., Thuy Binh, P., \& Dong, N. T. (2001). NMR investigation of chitosan derivatives formed by the reaction of chitosan with levulinicacid. Carbohydrate Polymers, 46(4), 339-348. http://dx.doi.org/10.1016/S01448617(00)00333-7.

20. Brugnerotto, J., Lizardi, J., Goycoolea, W., Argüelles-Monal, W., Desbrières, J., \& Rinaudo, M. (2001). An infrared investigation in relation with chitin and chitosan characterization. Polymer, 42(8), 3569-3580. http://dx.doi.org/10.1016/S00323861(00)00713-8.

21. López, F. A., Mercê, A. L. R., Alguacil, F. J., \& López-Delgado, A. A. (2008). A kinetic study on the thermal behaviour of chitosan. Journal of Thermal Analysis and Calorimetry, 91(2), 633-639. http://dx.doi.org/10.1007/s10973-007-8321-3.

22. Silva-Júnior, A. A., Scarpa, M. V., Pestana, K. C., Mercuri, L. P., Matos, J. R., \& Oliveira, A. G. (2008). Thermal analysis of biodegradable microparticles containing ciprofloxacin hydrochloride obtained by spray drying technique. Thermochimica Acta, 467(1-2), 91-98. http://dx.doi.org/10.1016/j.tca.2007.10.018.

23. Peng, Y., \& Li, Y. (2014). Combined effects of two kinds of essential oils on physical, mechanical and structural properties of chitosan films. Food Hydrocolloids, 36, 287-293. http:// dx.doi.org/10.1016/j.foodhyd.2013.10.013.

24. Liu, M., Zhou, Y., Zhang, Y., Yu, C., \& Cao, S. (2014). Physicochemical, mechanical and thermal properties of chitosan films with and without sorbitol. International Journal of Biological Macromolecules, 70, 340-346. http://dx.doi. org/10.1016/j.ijbiomac.2014.06.039. PMid:24984024.

25. Leceta, I., Arana, P., Guerrero, P., \& de la Caba, K. (2014). Structure-moisture sorption relation in chitosan thin films. Materials Letters, 128, 125-127. http://dx.doi.org/10.1016/j. matlet.2014.04.123.

Enviado: Abr: 07, 2014

Revisado: Set. 15, 2014 Aceito: Jan. 13, 2015 